\title{
Hormone and veterinary drug residue analysis in food, feed, biological and environmental matrices
}

\author{
Siska Croubels ${ }^{1} \cdot$ Els Daeseleire $^{2} \cdot$ Sarah De Saeger $^{3}$ • Peter Van Eenoo ${ }^{4}$. \\ Lynn Vanhaecke ${ }^{5}$
}

Published online: 17 April 2015

(C) Springer-Verlag Berlin Heidelberg 2015

For decades the use of growth-promoting agents and veterinary drugs has been the subject of many international debates, leading to European Council Regulation (EEC) No. 2377/90 introducing maximum residue limits (MRLs) of veterinary medicinal products in food of animal origin. That regulation was subsequently repealed and replaced by Regulation (EC) No. 470/2009. All the pharmacologically active substances that have been assessed in accordance with this regulation are listed and classified in Commission Regulation (EU) No. 37/ 2010. Currently, 641 pharmacologically active authorized substances and nine prohibited substances are listed. In

Published in the topical collection on Hormone and Veterinary Drug Residue Analysis with guest editors Siska Croubels, Els Daeseleire, Sarah De Saeger, Peter Van Eenoo, and Lynn Vanhaecke.

Siska Croubels

siska.croubels@ugent.be

1 Department of Pharmacology, Toxicology and Biochemistry, Laboratory of Pharmacology and Toxicology, Faculty of Veterinary Medicine, Ghent University, Salisburylaan 133, 9820 Merelbeke, Belgium

2 Technology and Food Science Unit - Food Safety, Institute for Agricultural and Fisheries Research, Brusselsesteenweg 370, 9090 Melle, Belgium

3 Department of Bioanalysis, Laboratory of Food Analysis, Faculty of Pharmaceutical Sciences, Ghent University, Ottergemsesteenweg 460, 9000 Ghent, Belgium

4 Doping Control Laboratory (DoCoLab), Faculty of Medicine and Health Sciences, Ghent University, Technologiepark 30, 9052 Zwijnaarde, Belgium

5 Department of Veterinary Public Health and Food Safety, Laboratory of Chemical Analysis, Faculty of Veterinary Medicine, Ghent University, Salisburylaan 133, 9820 Merelbeke, Belgium addition, food additives with a valid $E$ number which are approved for human consumption are classified as "No MRL required". This implies residue surveillance monitoring in food and feed using appropriate analytical methods. These methods may be subdivided into screening methods and confirmatory methods. Similar methods are needed to analyze residues of these compounds in environmental samples and for anti-doping analysis in sports.

\section{Biosensors, rapid screening, and confirmatory assays}

For antimicrobial drug residues, screening analyses can be performed using microbiological inhibitor tests, ELISAs, receptor tests, and easy to use diagnostic kits. Over the last decade, especially in the milk-control sector, new developments have resulted in a substantial reduction in analysis time to less than $5 \mathrm{~min}$, a broadening of the range of compounds detected (e.g. multi-class antibiotic testing), an improved detection capacity in relation to MRL legislation, and simplified test design (e.g. combination of incubation and reading; suitability for use at ambient temperature).

Another development is the use of molecularly imprinted polymers (MIPs), some of which are commercially available. In most cases MIPs are used as the selective adsorbent in solid-phase extraction methods for the clean-up of samples. New electrochemical and optical immunosensors, flow-cytometry immunoassays, and biochip-array technology are already, or will soon be, available for residue analysis.

Despite its cost, during recent years mass spectrometry (MS) detection has been more frequently used as the screening 
technique in residue analysis. For targeted analysis of residues of veterinary drugs in one analytical run (multi-residue methods), liquid chromatography coupled to low-resolution tandem mass spectrometry (LC-MS-MS) is often the method of choice. The use of LC-MS-MS is common practice in confirmatory analysis and in drug-disposition and residuedepletion studies of antimicrobial drugs, anthelmintics, (non)steroidal anti-inflammatory drugs, coccidiostats, sedatives, and other pharmacologically active substances. Currently high-resolution mass spectrometry (HRMS), offering the advantages of a "non a priori" and retrospective approach and the possibility of performing $\mathrm{MS}^{\mathrm{n}}$, is beginning to be used in residue analysis. By building databases of known compounds, biological samples can be screened for the presence of those compounds. Additionally, these data can be used to identify possible unknowns, including phase I and phase II metabolites of known compounds. Indeed, in some cases not only the parent (original) substances have to be detected but also one or more metabolites, depending upon the matrix analyzed.

As well as the use of LC-MS-MS for residue analysis in food and feed, its application in ecotoxicology is also common practice. The untargeted approach of HRMS for screening for the presence of a virtually unlimited number of smallmolecule residues and contaminants in environmental samples has huge advantages in studying their environmental transfer and fate, and is expected to develop further advantages in the near future.

\section{Advanced mass spectrometry}

Since the beginning of residue analysis (ca 45 years ago), the main objective has been to improve analytical methods by lowering decision limits $(\mathrm{CC} \alpha)$ and detection capabilities $(\mathrm{CC} \beta)$, increasing the number of analytes, shortening run times, increasing sample throughput, and other methods. Technological developments in high-end instruments, in particular in mass spectrometry, have supported this evolution. To ensure that decisions are based on sound science and evidence, it is of paramount importance that these techniques are assessed in terms of selectivity. This is true both for the more established MS-MS technique and for stand alone and hybrid HRMS technology. In Commission Decision (CD) 2002/657/EC the technical guidelines for the performance characteristics, limits, and criteria for confirmatory methods are described. To guarantee the identification of a compound the concept of identification points and ion ratios was introduced. It is also mentioned that for HRMS the resolution has to be $>10,000$ for the whole mass range. However, taking into account the technical evolution of HRMS instrumentation, a critical re- assessment of CD 2002/657/EC and the proposal of new and/or amended robust performance characteristics for screening and confirmatory analysis are warranted. Aspects including resolution, mass accuracy, and absence of measurable background have to be considered. Also, with respect to chromatographic separation, many advances including ion mobility, liquid separation cells, hydrophilic interaction, and MIP column materials have been made in recent years. One additional important technology, with proved advantages for distinguishing endogenous substances from their synthetic copies, is isotope-ratio MS (IRMS).

\section{Omics and biomarker-based approaches}

Technological developments have led to the detection of previously unnoticed traces of xenobiotic substances (e.g. thiouracil, prednisolone, and chloramphenicol). Through the use of metabolomics (relying on MS-MS, HRMS, and hybrid HRMS systems), untargeted fingerprinting of biological matrices may enable marker molecules to be proposed to discriminate between the endogenous prevalence and exogenous administration of these xenobiotics. A similar method may be used for proving illegal use of natural steroids in livestock production (steroidome profiling), because administration of steroids is known to induce temporal modifications of endogenous steroid profiles. One challenge that remains with respect to newly identified biomarkers is to free the established statistical models from their respective experimental conditions, to develop models able to make predictions regarding samples arising from other experimental conditions, i.e. other animals (age, sex, and breeding conditions), and dealing with other compounds, doses, and treatment lengths. Also, analysis of the transcriptome has been revealed as a promising approach to discover the unauthorized use of anabolic steroids on the basis of physiological changes. Proteomics approaches may also be used to, e.g., identify plasma proteins indicative of the biological response of livestock after treatment with different steroids or corticosteroids.

\section{Current and future evolution of anti-doping analysis}

Anti-doping analysis has evolved from the analysis of urine samples for a limited number of substances using radioimmunoassays (RIA) and gas chromatography in the $70 \mathrm{~s}$ and $80 \mathrm{~s}$, towards a much more comprehensive screening of both blood and urine using a wide range of technology. Recently, the use of internet databases 
has enabled links to be established between samples from a single athlete without jeopardizing the anonymity of the athlete to the laboratory. Hence, for endogenous substances, decisions on the normality or abnormality of observed concentrations or ratios can be on the basis of individual limits rather than population-based limits. The development and consequent use of peptide and proteinbased drugs has led to the need to diversify both matrices and analytical techniques. This trend will continue in the near future and will first lead to the use of more immunology-based detection technology, which will gradually be replaced by chromatography-MS techniques.

In a similar manner to the replacement of RIA with GCMS in the 80 s, LC-MS techniques are now gradually replacing the oldest immunoassay-based techniques for peptide doping. Human chorionic gonadotrophin, insulin-like growth factor 1, darbepoetin-alpha, and growth-hormone-releasing peptides can now be detected by LC-MS.

As the nature of doping agents expands to new classes of chemical structure, new technology is needed, but new matrices can also be introduced. Ethical considerations prevented the collection of blood samples until the last decade. Currently blood is a well-established additional matrix, and it is clear that for some substances hair, saliva, or sweat might offer advantages.

For the analytical chemist, however, there are additional opportunities which are yet to be realized. The introduction of novel technology (e.g. GC-MS-MS, LC-MS-MS, and LC-HRMS) has often resulted in the transfer of existing methods without full reconsideration. Often a change in "end pipe" technology can lead to substantial changes to the whole method, and it is expected that in the near future these will be investigated. Examples include the detection of intact phase II metabolites with LC-MS (whereas previously deconjugation was required for GC-MS and LC-MS) or the use of chemical ionization (CI) in GC-MS-MS (whereas electron ionization (EI) was previously regarded as the method of choice).

It is clear that these fields and techniques of residue analysis in food, feed, biological, and environmental matrices will be fully investigated over the coming years and will result in much innovation in this particular field of bioanalytical chemistry. In this regard, the international symposium series on Hormone and Veterinary Drug Residue Analysis, founded in 1988 and since then known as the "Ghent Residue Meeting", offers an excellent venue for presentation and discussion of the latest scientific and instrumental advances in residue analysis (www.vdra.ugent.be/).

We would like to thank all authors for their outstanding contributions, and also the editorial office of Analytical and Bioanalytical Chemistry for the professional collaboration.

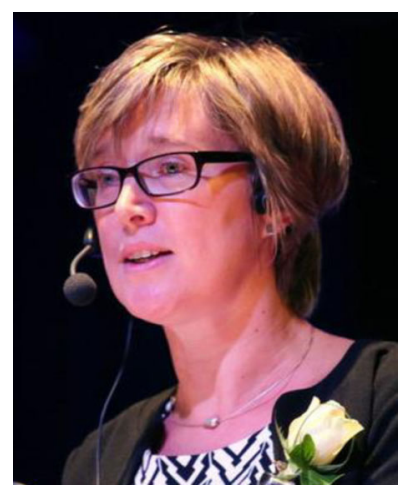

Siska Croubels is Full Professor and Head of the Laboratory of Pharmacology and Toxicology at the Faculty of Veterinary Medicine of Ghent University in Belgium. She is an Associate Member of the European College of Veterinary Pharmacology and Toxicology. Her laboratory has experience in the analysis of drugs, mycotoxins and metabolites in biological samples, using mainly (U)HPLC-MS-MS. Her research group in toxicology is member of the MYTOX platform (www.mytox.be/), dealing with the analysis and effects of mycotoxins on animal health. The research in pharmacology relates to the pharmacokinetics, pharmacodynamics and residues of veterinary drugs in several animal species; and to the development of suitable animal models for drug research.

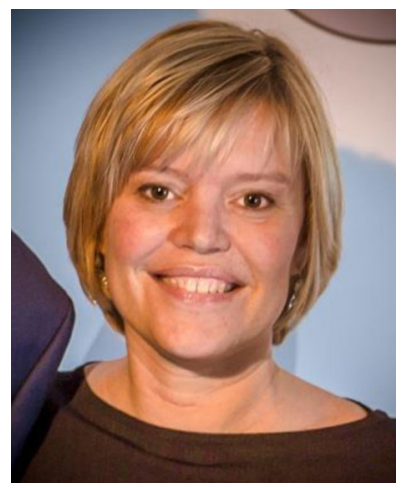

Els Daeseleire is Group Leader $(\mathrm{PhD})$ in chemical food safety at the Institute for Agricultural and Fisheries Research in Melle, Belgium. In collaboration with Dr. P. Delahaut (CER), since January 2013 she has been responsible for the national reference laboratory residues in Belgium. She has particular experience in the development and validation of LC-MSMS methods for the detection of residues of veterinary drugs and mycotoxins in biological matrices. In the last year LC-HRMS

has also been introduced in to the laboratory and new horizons have been explored to extend the expertise to the analysis of plant materials (plant hormones, polyphenols, ...).

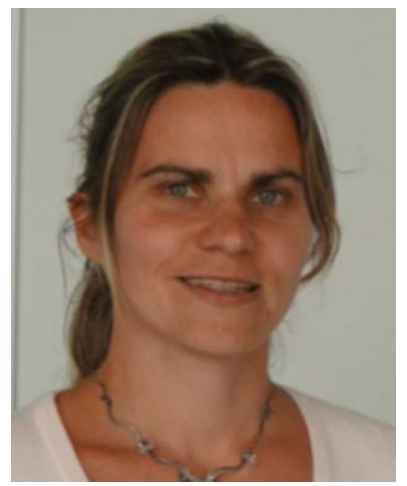

Sarah De Saeger is Professor and Director of the Laboratory for Food Analysis at the Faculty of Pharmaceutical Sciences at Ghent University in Belgium. Her research area includes the development of new technologies for analysis (screening and confirmation) of mycotoxins in food, feed and biological samples. Current focus is on mycotoxin global surveys, unknown fungal metabolites, and biomarkers of human exposure. She is coordinator of the MYTOX platform (www.mytox.be/). 


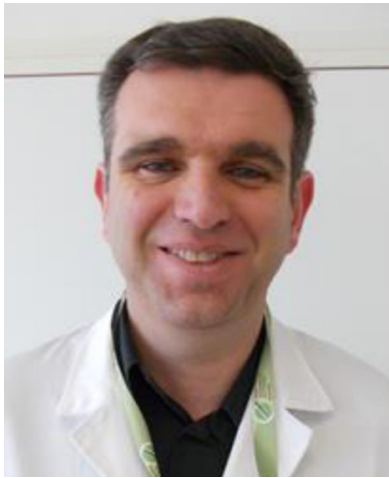

Peter Van Eenoo is Full Professor and Head of the WADAaccredited Doping Control Laboratory at the Faculty of Medicine and Health Sciences of Ghent University in Belgium. Currently, he is President of the World Association of Anti-Doping Scientists, representing 34 WADA accredited and probationary laboratories. $\mathrm{He}$ was active as a scientific expert in different laboratories at the occasion of several Olympic Games. His research mainly focuses on the metabolism of doping agents and analysis of these substances using GC-MS and LC-MS methodologies. Additionally, he has a special interest in quality assurance.

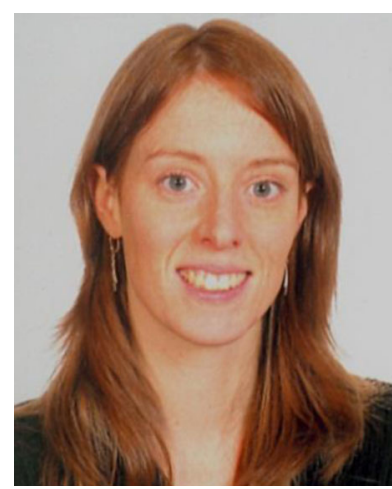

Lynn Vanhaecke is Professor of Food and Environmental Analytical Chemistry and Director of the Laboratory of Chemical Analysis at the Faculty of Veterinary Medicine of Ghent University in Belgium. Her research area includes the analysis of residues and contaminants in food and environmental matrices, the chemical analyses and biological activity of food constituents, and the metabolomic profiling of biological, food and environmental matrices. To this extent, advanced mass spectrometric techniques and in particular HRMS are her core expertise. 